\title{
Valorisation des ordures ménagères sous forme énergétique
}

\author{
N. Hafid et M. El Hadek \\ Laboratoire de Chimie Minérale Appliquée et Génie des Procédés, Université Ibn Zohr, \\ Faculté des Science, BP. 28/S, Agadir 80000, Morocco
}

\begin{abstract}
The household refuse of Agadir city have a high level of moisture and contain large amount of organic materials witch can be used in agriculture. Nevertheless, the plastic waste account for about $10.7 \%$ percent by weight of domestic waste and poses an inconvenient to physical characteristics of the municipal solid waste compost. This work aims to sort by resin type various plastics packaging by using differential scanning calorimetry and to evaluate their heat of combustion by thermometric calorimetry. Experimental results chow a significant part of polyethylene containers that can be recycled. The plastics with very small volume chow a very heterogeneous composition but have a high heat value, approximately two times greater than to heat released by wood. Plastics burn with very little ash but most environmental concerns associated with their combustion have been related to gas emissions. Those are analysed by gas chromatography.
\end{abstract}

\section{INTRODUCTION}

La progression de la consommation des emballages ménagers en matières plastiques s'est répercutée sur la nature des ordures ménagères. D'après la Direction générale des collectivités locales [1], les matières plastiques représentent jusqu'à $45 \%$ des produits non fermentescibles dans les déchets ménagers marocains. Ces matières portent préjudice à la qualité du compost urbain et entrave sa commercialisation. Cette situation nous a amené à déterminer la valeur énergétique du compost urbain de la ville d'Agadir et à identifier, par voie calorimétrique, les différents plastiques prédominants dans les ordures ménagères ainsi qu'à déterminer leur chaleur de combustion et les gaz qu'ils engendrent.

\section{MATERIELS ET METHODES}

Les échantillons du compost sont prélevés à partir de dix andains de compost déposés sur l'aire de maturation au sein de l'unité de traitement des ordures ménagères de la ville. Les prises des échantillons bruts ont été effectuées à différents points de l'andain. Ils sont ensuite mélangés, homogénéisés et quartés. Leur composition physique est déterminée par la méthode de tri par classe. La fraction terreuse a été broyée finement puis analysée en triple. La méthode de walkly-black [2] a permis d'évaluer la teneur en carbone organique. Le taux des matières volatiles totales a été déterminé par calcination à $550^{\circ} \mathrm{C}$.

Les températures et les enthalpies de transition des différents plastiques sont mesurées avec le calorimètre enthalpique différentiel DSC7 Perkin Elmer. Les mesures sont effectuées sous azote $U$ avec un débit de $20 \mathrm{ml} / \mathrm{min}$ et une vitesse de chauffe de $5^{\circ} \mathrm{C} / \mathrm{min}$.

Les chaleurs de combustion ont été évaluées par utilisation du calorimètre à bombe d'oxygène Parr 1341. Les plastiques et le compost ont été préparés sous forme de pastilles et leur combustion s'est déroulée sous une atmosphère d'oxygène à pression de 35 bars puis sous air à différentes pressions. Les gaz de combustion ont été analysés par chromatographie en phase gazeuse à détecteur FID. 


\section{RESULTATS ET DISCUSSION}

Dans la ville d'Agadir, les activités domestiques génèrent quotidiennement 250 tonnes d'ordures ménagères. Les matières putrescibles représentent $77 \%$ et les matières plastiques $10.7 \%$ du poids total [3]. Ce dernier taux dépasse largement la moyenne nationale [1]. Le choix adopté dans l'unité de traitement des ordures ménagères est le compostage en andains retournés à l'air libre. Le tri manuel du produit final montre qu'il est constitué de $10 \%$ de contaminants physiques dont $1.4 \%$ est relatif au plastique. Celui-ci porte préjudice à l'aspect visuel du compost et entrave son écoulement.

L'analyse chimique des échantillons de compost issus de dix andains montre qu'il est riche en matières organiques. En effet, les teneurs en matières volatiles totales à $550^{\circ} \mathrm{C}$ varient entre 37 et $47 \%$ et celles du carbone organique assimilable entre 10 et $15 \%$ de la matière sèche (tableau I).

Le pouvoir calorifique (P.cal) des différents échantillons se situe entre 1200 et $2200 \mathrm{cal} / \mathrm{g}$. Cette valeur énergétique est supérieure à celle des composts urbains français [4] mais reste néanmoins inférieure à celle des combustibles traditionnels. La détermination de la participation de la valeur énergétique du plastique dans celle du compost et des refus de compostage nécessite une identification des résines de base des différents plastiques prépondérants dans les ordures ménagères brutes.

Tableau I : Teneurs en matières volatiles totales, en carbone organique et le pouvoir calorifique du compost

\begin{tabular}{l|rrrrrrrrrr}
\hline$N^{\circ}$ Andain & 1 & 2 & 3 & 4 & 5 & 6 & 7 & 8 & 9 & 10 \\
\hline$\%$ M.V.T & 40.28 & 44.33 & 41.63 & 42.40 & 43.09 & 43.87 & 46.75 & 45.02 & 40.91 & 37.11 \\
& & & & & & & & & & \\
\% Corg $(\% \mathrm{MS})$ & 11.14 & 14.13 & 14.13 & 15.03 & 13.53 & 12.81 & 14.81 & 9.93 & 10.80 & 10.31 \\
& & & & & & & & & & \\
P.cal (cal/g) & 2261 & 1730 & 1696 & 1752 & 1276 & 2109 & 2244 & 1709 & 1938 & 1455 \\
\hline
\end{tabular}

L'analyse thermique par calorimétrie différentielle à balayage des différents plastiques a montré que dans la série des corps creux, la forme la plus répandue est à base du polyéthylène. La superposition de quelques spectres obtenus à celui du polyéthylène haute densité est représentée sur la figure 2. L'analyse a montré aussi que seules les bouteilles en plastique des eaux minérales et de certaines boissons gazeuses sont à base du polyéthylène téréphtalates. L'évolution de la température lors de leur combustion est variable comme le montre la figure 2 . Pour ces différents plastiques, les chaleurs de combustion sont légèrement inférieures à celle des résines de base.

Les sachets et les sacs en plastiques sont d'une grande diversité et connaissent une consommation croissante [5]. Leur légèreté et leur faible taille rendent leur récupération difficile. L'analyse thermique de ces plastiques a montré une hétérogénéité en composition et un pouvoir énergétique variable mais il atteint pour une grande partie $43 \mathrm{KJ} / \mathrm{g}$ soit deux fois celui du charbon du bois. En effet, cette grandeur dépend de plusieurs paramètres dont la composition, l'humidité des déchets et le mode suivi pour les collecter [6].

La combustion des plastiques sous air dans la bombe à oxygène a permis de prélever les gaz résultants. Leur analyse par chromatographie en phase gazeuse montre des faibles teneurs en gaz organiques. 


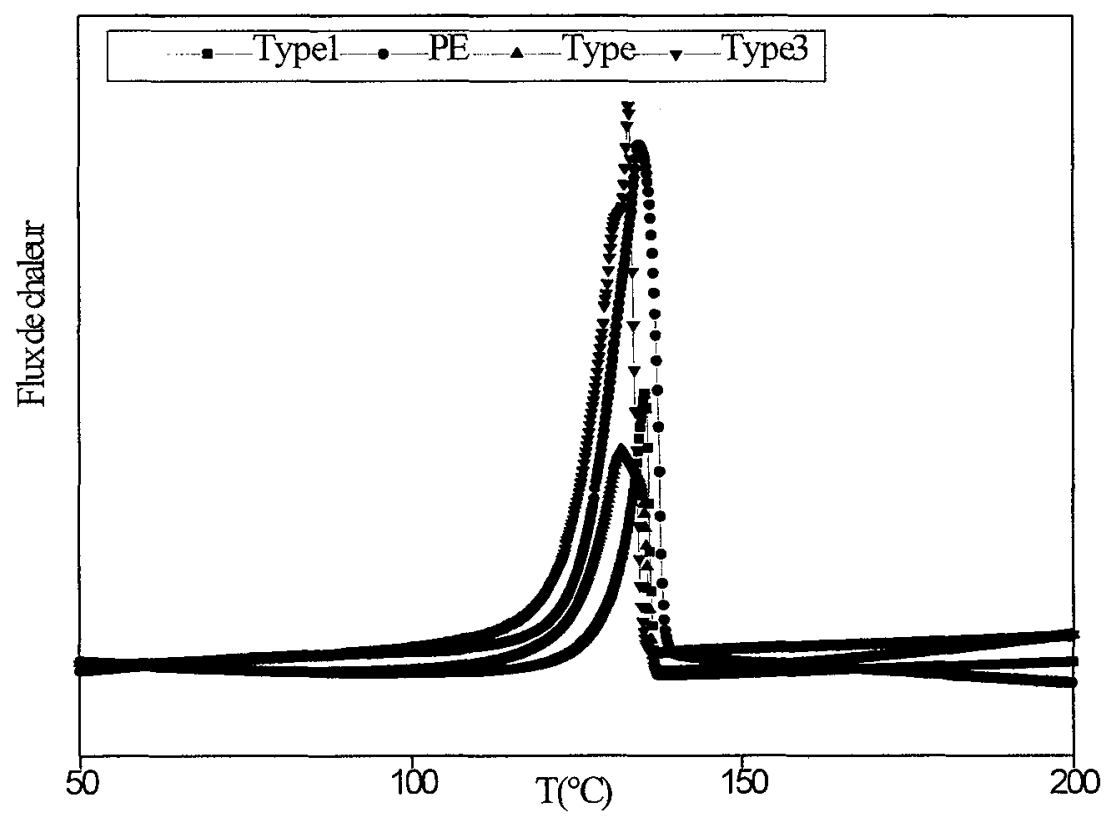

Figure 1. Spectres DSC de quelques emballages plastiques à base du polyéthylène haute densité

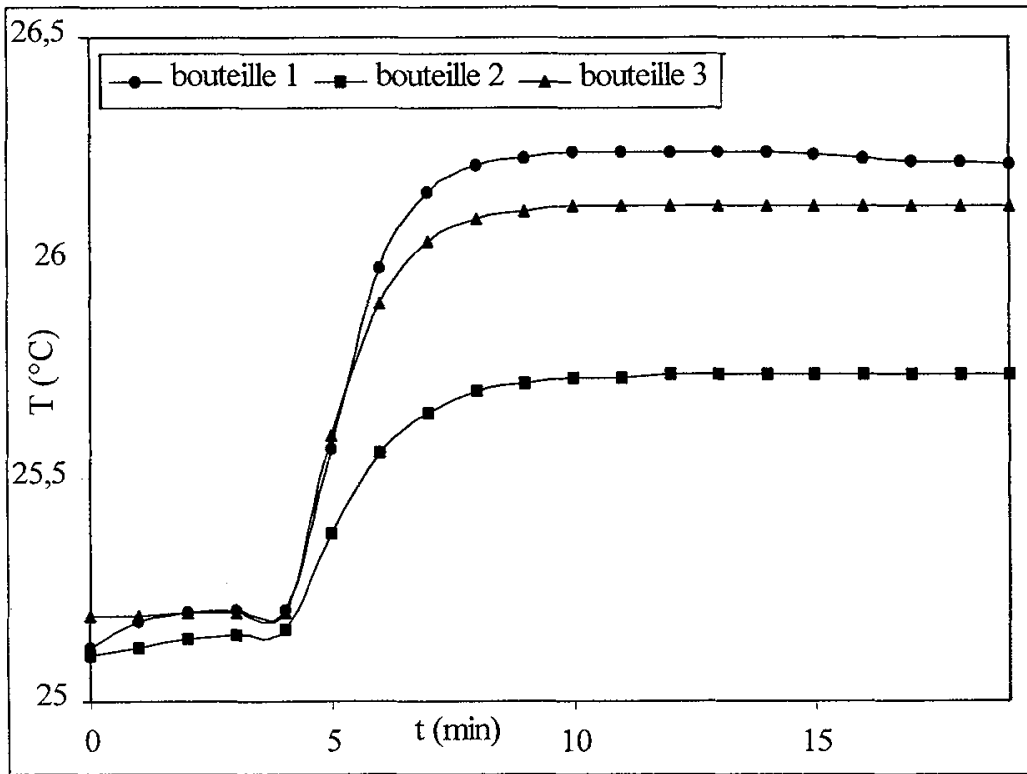

Figure 2. Evolution de la température lors de la combustion des bouteilles en polyéthylène téréphtalates 


\section{CONCLUSION}

Les ordures ménagères du Maroc sont humides et riches en matières organiques. Le tri à la source de ces déchets permet de valoriser la fraction putrescible sous forme de compost de qualité. Il permet aussi une réinsertion plus facile des matières plastiques dans le circuit industriel. Vu l'absence d'unités d'incinération et le coût élevé de leur mise en place, il serait judicieux que les emballages ménagers à base de polyéthylène soient recyclés. Les sacs et les sachets peuvent être utilisés pour substituer une part des combustibles traditionnels.

\section{Références}

[1] GERSAR/SCP. ANRED-ADEME, Collecte et traitement des ordures ménagères au Maroc, Dir. Gén. Coll. Loc. (1995) pp. 1-48.

[2] Segoe S. Rd., Methods of Soil Analysis, Agronomy Monograph n ${ }^{\circ}$ 9, Part 2. Chemical and Microbiological Properties, $2^{\text {nd }}$ Ed. ASA, Madison, (1982).

[3] Lguirati A., Thèse de $3{ }^{\text {ème }}$ cycle-Agadir, (1998) pp. 1-106.

[4] Jemali B., Thèse de $3{ }^{\text {eme }}$ cycle - Eljadida, (1996) pp. 1-127.

[5] Ministère de l'aménagement du territoire, de l'environnement, de l'urbanisme et de l'habitat, Etude pilote sur les sacs et sachets en plastique au Maroc, Rapport final, pp. 1-124.

[6] Maystre L.Y. et al. , Déchets urbains Nature et caractérisation, Gérer l'environnement, 1 ière Ed. CH - 1015 Lausanne, Suisse (1994) pp.1-219. 